\title{
Therapeutic effects of bee venom on experimental atopic dermatitis
}

\author{
HYEMIN GU $^{1 *}$, WOON-HAE KIM ${ }^{1 *}$, HYUN-JIN AN $^{1}$, JUNG-YEON KIM ${ }^{1}$, \\ MI-GYEONG GWON ${ }^{1}$, SANG MI HAN ${ }^{2}$, JAECHAN LEEM ${ }^{3}$ and KWAN-KYU PARK ${ }^{1}$ \\ ${ }^{1}$ Department of Pathology, School of Medicine, Catholic University of Daegu, Daegu 42472; \\ ${ }^{2}$ Department of Agricultural Biology, National Academy of Agricultural Science, Wanju, Jeollabuk-do 55365; \\ ${ }^{3}$ Department of Immunology, School of Medicine, Catholic University of Daegu, Daegu 42472, Republic of Korea
}

Received March 22, 2018; Accepted July 20, 2018

DOI: $10.3892 / \mathrm{mmr} .2018 .9398$

\begin{abstract}
Atopic dermatitis (AD) is a chronic skin inflammatory disease characterized by recurrent eczema and itching. It is caused by a poorly controlled immune response and damage to the skin barrier. Purified bee venom (BV) is a natural toxin produced by honeybees (Apis mellifera L.), and is well known for its anti-inflammatory, analgesic and anti-cancer effects against various types of disease. However, treatment strategies based on anti-inflammatory properties have not been adequately studied in AD. Thus, the present study examined the progression of AD-like skin lesions induced by ovalbumin (OVA) and the mechanism of action of BV. BV, administered by intraperitoneal inoculation, was observed to reduce the symptoms of $\mathrm{AD}$, in addition to the serum immunoglobulin E levels, according to dorsal skin thickness and histopathologic analysis. The treatment also inhibited the infiltration of eosinophils and mast cells. These results suggested that it is possible to develop novel AD alternative therapy using BV by effectively suppressing allergic skin inflammation in AD.
\end{abstract}

\section{Introduction}

Atopic dermatitis (AD) is a common chronic inflammatory and pruritic skin disease characterized by eczematous, dry, and chapped skin $(1,2)$. AD is caused by the invasion of inflammatory cells such as the mast cells, eosinophils, monocytes/macrophages and $\mathrm{T}$ lymphocytes into the skin barrier (3). AD is also reported that the development is

Correspondence to: Professor Kwan-Kyu Park, Department of Pathology, School of Medicine, Catholic University of Daegu, 33 Duryugongwon-ro 17-gil, Nam-gu, Daegu 42472, Republic of Korea

E-mail: kkpark@cu.ac.kr

${ }^{*}$ Contributed equally

Key words: atopic dermatitis, bee venom, anti-inflammatory skin lesion increased levels of circulating eosinophils and serum immunoglobulin E ( $\mathrm{IgE}$ ) owing to elevated manufacture of interleukin (IL)-4, IL-5, and IL-13 by the Th2 cells in most patients $(4,5)$. IgE plays an important role in instantaneous hypersensitivity responses by cross-linking to the high-affinity IgE receptor (FceRI) expressed on the mast cells and basophils. The antigen-specific IgE binding to FcRI is the binding of multivalent antigens of allergens that contribute to allergic diseases, leading to the release of inflammatory mediators such as histamine, arachidonic acid metabolites and cytokines by activating mast cells $(4,5)$.

Mast cells, inflammatory cells that respond to innate and adaptive immunity, can be described as allergic effector cells that secrete numerous cytokines associated with chronic skin inflammation $(6,7)$. They induce the production of inflammatory mediators, such as histamine, chemokines, and cytokines, under degranulation and immunostimulation (8). These inflammatory mediators significantly influence the development of AD (9). It has been demonstrated in several studies that thymic stromal lymphopoietin (TSLP) is associated with the development, maintenance, and enhancement of AD (10-12). It has been identified as an activation factor for itching; a characteristic of AD. TSLP plays a critical role in the activation of dendritic cells that prime human CD4+ T cells as Th2 cytokine-generating cells within the local lymph nodes (13-15). TSLP signaling in the CD4+ T cells is required for the formation of memory following Th2 sensitization (16). It activates the innate lymphoid cells, a significant players in the pathogenesis of several inflammatory skin diseases, including AD (17).

Filaggrin plays an important structural and functional role in the epidermis, and has a significant effect on skin homeostasis (18). It is instrumental in maintaining skin hydration, by preserving the integrity of the stratum corneum, and in the production of natural moisturizing factors (19). Inherited or acquired filaggrin deficiency has been described as a key contributor to the pathogenesis of AD (18). Thus, the inhibition of production of these components constitutes a promising and important modality in the treatment of patients with allergic disease, especially those with AD.

A number of treatments are available for AD, including the use of emollients, dexamethasone, topical glucocorticoids, 
calcineurin inhibitors, and immunosuppressants, such as cyclosporine A; all of which are effective in reducing inflammation. However, they are associated with various side-effects $(1,2)$. Therefore, the need for the development of new pharmacological agents that control the inflammatory responses, with reduced side-effects and higher efficacy, has been identified.

Bee venom (BV) is a natural toxin produced by honeybees (Apis mellifera L.). BV contains various peptides including melittin, apamin, adolapin, and mast cell degranulating peptide along with enzymes, biological amines, and non-peptide components (20). BV is widely used as a traditional medicine for various diseases $(20,21)$. The treatment of inflammatory diseases has been investigated in several studies (22-24). The anticancer properties of BV have also been demonstrated in lung and breast cancer, hepatocellular carcinoma, and prostate cancer cells (25-27). In previous study, we demonstrated that melittin of main BV components has beneficial effects on atopic-dermatitis (28). However, the anti-inflammatory effects of BV on ovalbumin (OVA)-induced inflammatory skin disease in an animal model have not been reported to date. Thus, the current study objective was to evaluate the therapeutic effects of BV as an alternative to anti-inflammatory therapy for the treatment of $\mathrm{AD}$.

\section{Materials and methods}

$B V$ collection. Animal care and all experimental procedures were approved by the Institutional Animal Care and Use Committee of Catholic University of Daegu (Daegu, Korea; approval no. DCIAFCR-160428-1-Y) and experiments were performed in accordance with these institutional guidelines. The natural honeybee colonies used in the present study were maintained at the National Academy of Agricultural Science (Suwon, Korea). BV was collected with the aid of a collecting device (Chung Jin Biotech Co., Ltd., Ansan, Korea) in a sterile manner under strict laboratory conditions. In brief, the BV collector device was placed in the hive and the bees were administered electric shocks to caused them to sting a glass plate from which dried BV was later removed by scraping. The collected venom was purified using the method described by Han et al (29) The BV was then stored in a refrigerator for later use.

Animals and induction of $A D$. Six-week-old female BALB/c mice $(n=25)$ were purchased from Samtako (Osan, Korea) and housed at $22 \pm 2^{\circ} \mathrm{C}$ and $55 \%$ humidity in a $12 \mathrm{~h}$ light-dark cycle, and allowed food and water ad libitum. The mice were equilibrated for seven days prior to the induction of $\mathrm{AD}$ and randomly divided into five groups (5 mice per group). These were divided into 5 groups as follows: the normal control (NC), OVA-induced AD with no treatment (OVA), and OVA-induced AD with various concentrations $(1,10$ and $100 \mu \mathrm{g} / \mathrm{kg}$ of weight) of BV-treatment (BV1, BV10 and BV100).

Induction of $\mathrm{AD}$ was performed according to the method given by Kim et al (28) Briefly, All the mice, with the exception of those in the NC group, were intraperitoneally inoculated mixed with $10 \mu \mathrm{g}$ of chicken OVA (grade V) (Sigma-Aldrich; Merck KGaA, Darmstadt, Germany) mixed with $4 \mathrm{mg}$ of aluminum hydroxide (ImjectAlum ${ }^{\circledR}$; Thermo Fisher Scientific, Inc., Waltham, MA, USA) in a volume of $200 \mu \mathrm{l}$ three time at intervals of one-week intervals (i.e., on days 0,7 , and 14). They were then anesthetized by isoflurane inhalation (Ifran ${ }^{\circledR}$; HANA Pharm, Seoul, Korea). During anesthesia, the dorsal skin was shaved. After shaving, the mice were sensitized with OVA patches on day 14. The OVA patches were prepared as $100 \mu \mathrm{g}$ of OVA in phosphate-buffered saline (PBS). The OVA patches were attached to the shaved skin for seven days (i.e., from days 14 to 20) and changed daily. Thereafter, the indicated concentration of $\mathrm{BV}(1,10$, and $100 \mu \mathrm{g} / \mathrm{kg}$ of weight) was administrated twice a week intraperitoneally. Following the BV treatment, the OVA patches were reattached for a week. After the OVA treatment with patches, their blood was collected by cardiac puncture, and the mice were sacrificed by $\mathrm{CO}_{2}$ asphyxiation. After sacrifice, their dorsal skins were excised for the next experiments.

Histologic analysis. The dorsal skin specimens were fixed in $10 \%$ neutral buffered formalin for at least $24 \mathrm{~h}$ at room temperature. The fixed skin specimens were dissected, dehydrated, and embedded in paraffin. Thereafter, thin sections $(4 \mu \mathrm{m})$ were mounted on glass slides and stained with hematoxylin and eosin (H\&E) and giemsa. All slides were examined under a Pannoramic ${ }^{\circledR}$ MIDI slide scanner (3DHISTECH Ltd., Budapest, Hungary). The number of infiltrated and degranulation mast cells was counted at magnification, $\mathrm{x} 400$ with $\mathrm{CaseViewer}{ }^{\circledR} 1.4$ software (3DHISTECH Ltd.).

Immunohistochemical staining. The paraffin-embedded tissue sections were deparaffinized with xylene, dehydrated in gradually decreasing concentrations of ethanol, and then subsequently treated with $3 \%$ hydrogen peroxidase in methanol for 10 min to block endogenous peroxidase activity. The tissue sections were immersed in $10 \mathrm{mM}$ sodium citrate buffer (a $\mathrm{pH}$ of 6.0) for $5 \mathrm{~min}$ at $95^{\circ} \mathrm{C}$. The final step was repeated using $10 \mathrm{mM}$ sodium citrate solution (a $\mathrm{pH}$ of 6.0 ). The sections in the same solution were left to cool at room temperature for $20 \mathrm{~min}$, rinsed in PBS, and then incubated with primary antibody (1:100 dilution) for $1 \mathrm{~h}$ at $37^{\circ} \mathrm{C}$. Anti-CD4 and anti-CD11b antibodies (Abcam, Cambridge, UK) were used as the primary antibody. The signal was visualized using an Envision System ${ }^{\circledR}$ (Dako; Agilent Technologies, Inc., Santa Clara, CA, USA) for $30 \mathrm{~min}$ at $37^{\circ} \mathrm{C} .3,3^{\prime}$-diaminobenzidine tetrahydrochloride (DAB) was used as the coloring reagent, and hematoxylin was used as a counter stain. The slides were inspected with a Pannoramic MIDI slide scanner and the integrated optical density was analyzed with the i-Solution DT software.

Immunofluorescence staining. The paraffin-embedded dorsal skin specimens were deparaffinized with xylene and dehydrated in gradually decreasing concentrations of ethanol. The tissue sections were subsequently placed in blocking serum (5\% bovine serum albumin in PBS) at room temperature for $1 \mathrm{~h}$. The primary antibody (1:100 dilution) was added followed by overnight incubation at $4^{\circ} \mathrm{C}$, and secondary antibody (1:200 dilution) was performed for $4 \mathrm{~h}$ at room temperature. The nuclei were stained with Hoechst $33342^{\circledR}$ solution for $20 \mathrm{~min}$. The antibodies included filaggrin (cat. no. ENZ-ABS181; Enzo Life Sciences, Lausen, Switzerland) and anti-rabbit-biotinylated secondary antibodies conjugated with fluorescein 
A

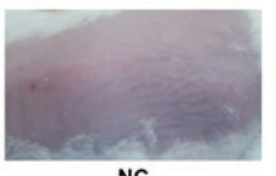

NC

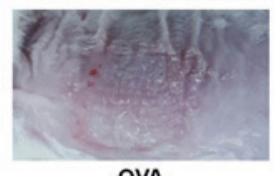

OVA

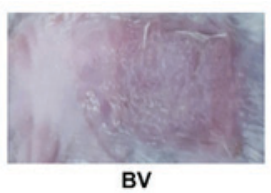

BV

B
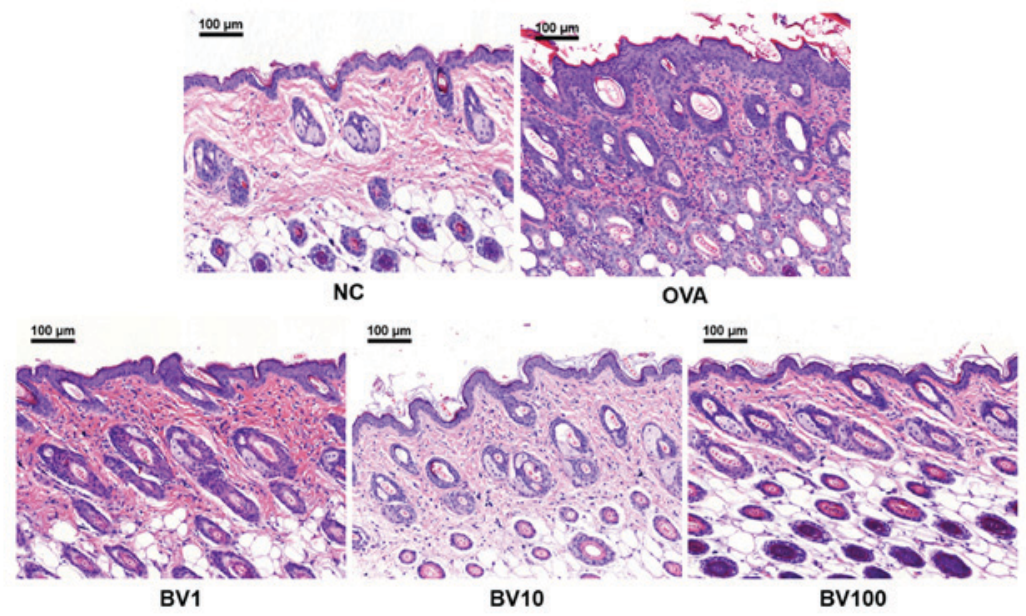

C

Epidermis
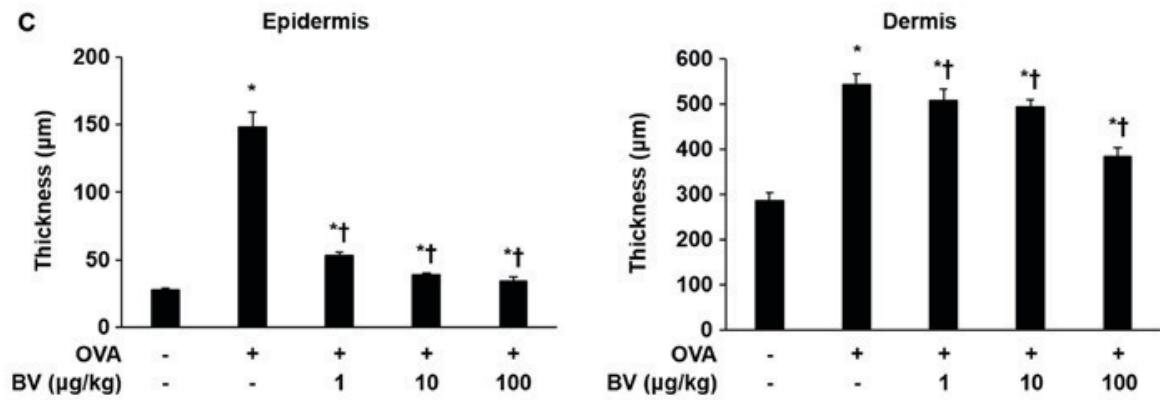

Figure 1. BV inhibits inflammation in OVA-induced inflammatory skin disease. OVA was used to induce atopic dermatitis-like inflammatory skin disease through intraperitoneal inoculation and patch attachment. BV was intraperitoneally inoculated during OVA induction. (A) Skin lesions of each group. (B) Hematoxylin and eosin staining: Representative images of histological analysis, exhibiting increased epidermal and dermal thickness, and increased number of invasive inflammatory cells in the dorsal skin specimens; however, this increase was reduced in the BV administration group. Magnification, $\mathrm{x} 200$; scale bars, $100 \mu \mathrm{m}$. (C) The thickness of the epidermis and dermis was measured. The results are expressed as the mean \pm standard error of the mean of least 10 random fields per section. ${ }^{*} \mathrm{P}<0.05$ vs. NC group; ${ }^{\dagger} \mathrm{P}<0.05$ vs. OVA group. BV, bee venom; OVA, ovalbumin; BV group, OVA with BV treatment (1, 10 and $100 \mu \mathrm{g} / \mathrm{kg}$ ); NC, normal control; OVA, ovalbumin; BV, bee venom.

isothiocyanate (Invitrogen; Thermo Fisher Scientific, Inc.). The slides were mounted using a fluorescence mounting medium (Dako; Agilent Technologies, Inc.). The stained slides were imaged using a $\mathrm{NIKON}^{\circledR} \mathrm{A} 1+$ confocal microscope (Nikon, Tokyo, Japan).

Enzyme-linked immunosorbent assay (ELISA). The concentrations of IgE, tumor necrosis factor (TNF)- $\alpha$, and TSLP in the collected sera of the blood were determined by ELISA kit, according to the manufacturer's instructions (IgE; Bethyl Laboratories, Montgomery, TX, USA) and R\&D Systems (TNF- $\alpha$ and TSLP). The optical density was measured at $450 \mathrm{~nm}$ using an ELISA reader (BMG Labtech, Baden-Württemberg, Germany).

Statistical analysis. All the data were presented as the mean \pm standard error of the mean. Statistical significance was determined by one-way analysis of variance with Tukey's multiple comparison test using GraphPad Prism 5.0 (GraphPad Software, Inc., La Jolla, CA, USA). P $<0.05$ was considered to indicate a statistically significant difference.

\section{Results}

Effect of BV treatment on dorsal skin thickness according to histopathologic analysis. As shown in Fig. 1A, the AD-like skin lesions induced by the OVA were clearly evident on the dorsal skin of the Balb/c mice. Various pathological features, such as bleeding, erythema, eczema, and dryness, were observed in the OVA group. By contrast, it was macroscopically confirmed that pathological features were significantly reduced in the BV group. Inflammatory cells infiltration and skin thickness were also measured in the epidermis and dermis of the mice skin by $\mathrm{H} \& \mathrm{E}$ staining (Fig. 1B and $\mathrm{C}$ ). As a result, infiltration by the inflammatory cells in the OVA group was significantly increased compared with that in the NC group, and the skin thickness was also significantly increased. By contrast, the extent of inflammatory cell infiltration and skin thickness was significantly decreased in the BV treatment group, and constituted the greatest decrease, especially in BV 100 group (Fig. 1C). Animal care and all experimental procedures were approved and implemented in accordance with the guidelines of the Institutional Animal 
A
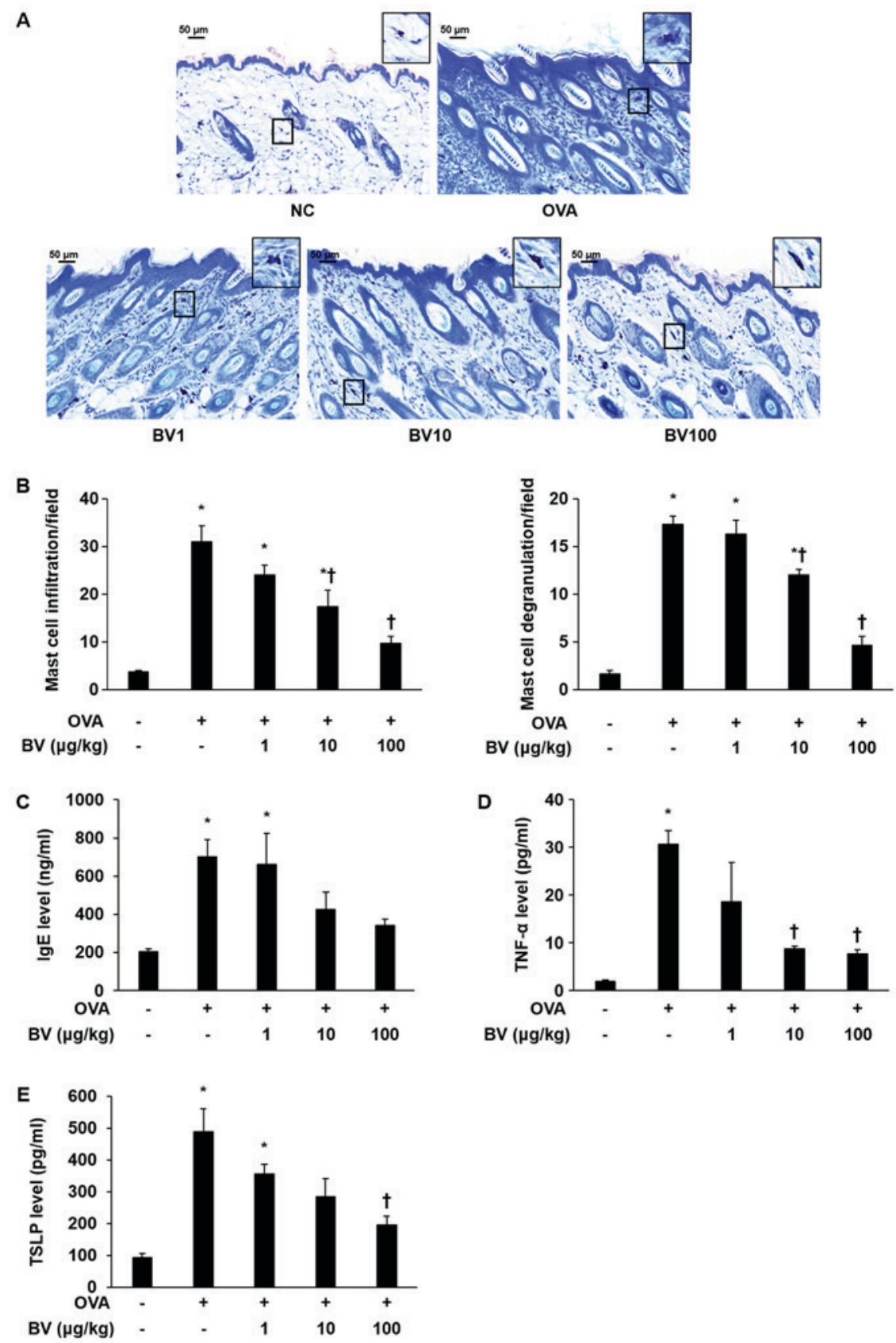

Figure 2. BV reduces OVA-induced mast cells, serum IgE and pro-inflammatory cytokine expression. (A) Giemsa staining: Representative images of histological analysis, presenting increased infiltration and degranulation of the mast cells. However, it was reduced under BV treatment. Magnification, x400; scale bars, $50 \mu \mathrm{m}$. (B) Infiltration and degranulation were evaluated by assessing the number of mast cells from at least 3 random fields per section at 400 -fold magnification. (C) The ELISA results demonstrated that BV inhibited the increased levels of serum-IgE induced by OVA. In addition, BV inhibited the increased expression of inflammatory cytokines such as (D) TNF- $\alpha$ and (E) TSLP induced by OVA. The results are expressed as the mean \pm standard error of the mean of 3 independent determinations. ${ }^{*} \mathrm{P}<0.05$ vs. NC group; ${ }^{\mathrm{P}} \mathrm{P}<0.05$ vs. OVA group. BV, bee venom; OVA, ovalbumin; BV group, OVA with BV treatment (1, 10 and $100 \mu \mathrm{g} / \mathrm{kg})$; NC, normal control; IgE, immunoglobulin; TNF- $\alpha$, tumor necrosis factor- $\alpha$; TSLP, thymic stromal lymphopoietin.

Care and Use Committee Catholic University of Daegu (approval no. DCIAFCR-160428-1-Y).

Effect of BV treatment on mast cell and IgE expression. To investigate the inhibitory effect of the BV treatment, infiltration and degranulation of mast cells was examined, and $\operatorname{IgE}$ levels were measured. The extent to which mast cell infiltration and degranulation occurred was measured at magnification, $\mathrm{x} 400$ (Fig. 2A). It was determined that the extent of mast cell infiltration and degranulation was significantly increased in the OVA group, but diminished in the BV treatment group, according to the concentration level applied. Notably, it was observed that the BV 100 group was significantly reduced (Fig. 2B). The escalation in the serum IgE level was shown to dramatically increased in the OVA group using ELISA. Similar to the measurement results of mast cell infiltration and degranulation extent, the serum IgE levels identified by ELISA were found to be reduced in the BV 100 group (Fig. 2C).

Effect of BV treatment on pro-inflammatory cytokine expression. TNF- $\alpha$ and TSLP levels were measured in the mouse serum of the $\mathrm{NC}$, OVA, and BV-treatment group to 

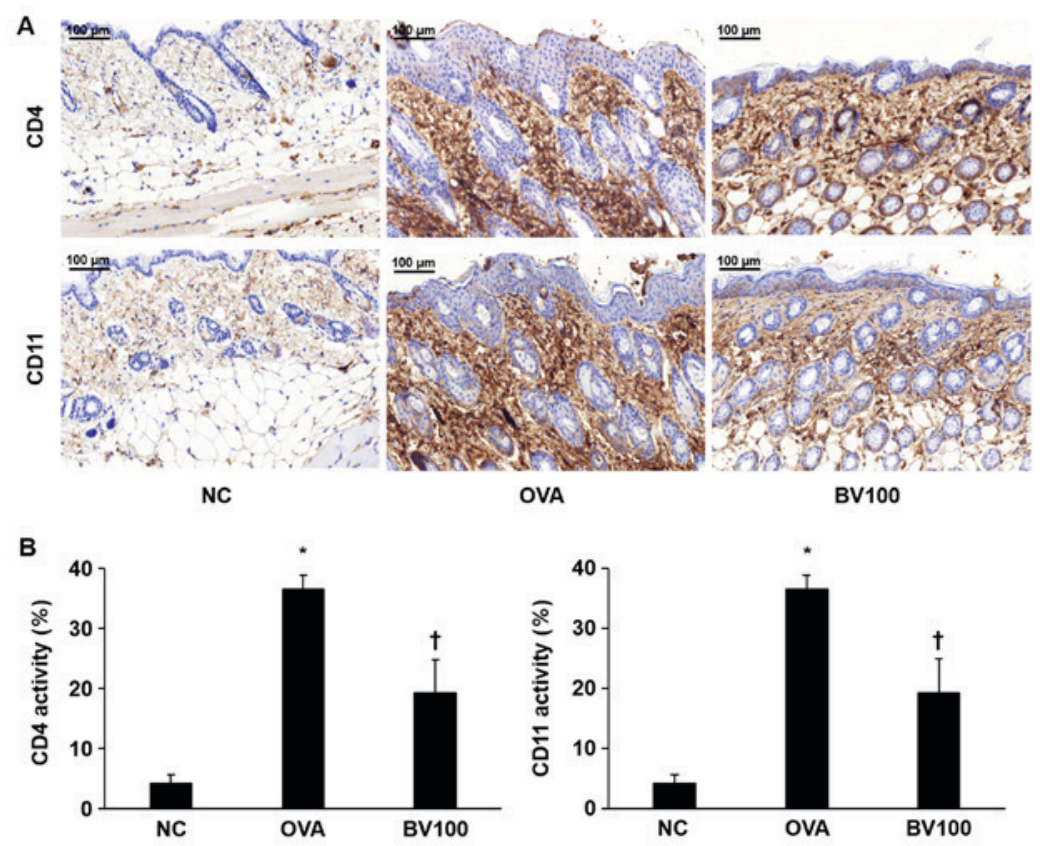

Figure 3. Expression of CD4+ and CD11b+ cells in the skin specimen. (A) The dorsal skin specimens were immunostained with CD4+ and CD11b+ antibodies Magnification, x200; scale bar, $100 \mu \mathrm{m}$. (B) The immunohistochemical results demonstrated that BV inhibits the expression of CD4+ and CD11b+. The integrated optical densities were measured from at least 3 random fields per section. The results are expressed as the means \pm standard error of the mean of 10 independent determinations. ${ }^{*} \mathrm{P}<0.05$ vs. NC group; ${ }^{\dagger} \mathrm{P}<0.05$ vs. OVA group. BV, bee venom; OVA, ovalbumin; BV group, OVA with BV treatment $(100 \mu \mathrm{g} / \mathrm{kg}) ; \mathrm{NC}$, normal control; CD, cluster of differentiation.

establish whether or not BV treatment would induce alterations to the pro-inflammatory cytokines involved in OVA-induced skin inflammation. The levels of TNF- $\alpha$ and TSLP were higher in the OVA-treated group than in the NC group. However, TNF- $\alpha$, and TSLP levels were significantly decreased in 100 group (Fig. 2D and E). IL-1 $\beta$ was reduced in BV100 but not statistically significant.

Effect of BV treatment on CD4+ and CD11b+expression. Immunohistochemical staining confirmed the extent of infiltration of the CD4+ T helper cells and CD11b+ known as monocyte antigen. As shown in Fig. 3A, the expression of CD4+ was significantly increased in the OVA group. On the other hand, the expression level of CD4+ was significantly decreased in the BV 100 group compared with OVA group. Similar to the results of the expression of CD4+, the expression level of CD11b+ was significantly increased in the OVA group, and it was also confirmed that the expression level of CD11b was also significantly decreased in the BV 100 group (Fig. 3B).

Effect of $B V$ treatment on filaggrin deficiency. Immunofluorescent staining was performed to determine the extent of filaggrin deficiency, which plays an important role in skin homeostasis. As shown in Fig. 4, the expression of filaggrin was strongly expressed in the NC group, but deficiency was confirmed in the OVA group. However, the expression of filaggrin was found to increase in the BV100 group.

\section{Discussion}

BV therapy has been used in Oriental medicine since ancient times to treat and relieve the pain associated with inflammatory diseases, such as rheumatoid arthritis and multiple sclerosis (21). Relatively BV of high concentrations $(\geq 2.5 \mu \mathrm{g} / \mathrm{ml})$ induce the release of inflammatory cytokines from the human keratinocytes (30). By contrast, BV of low concentrations $(\leq 100 \mathrm{ng} / \mathrm{ml})$ reduced pro-inflammatory cytokines in human keratinocytes infected with bacteria (31).

In previous studies, we reported that BV inhibits atherosclerotic lesions induced by an intraperitoneal injection of lipopolysaccharide (32). In addition, the in vitro antimicrobial activity of BV against inflammation induced by Propionibacterium acnes was demonstrated (33). However, the anti-inflammatory effects of BV on OVA-induced inflammatory skin disease in an animal model have not yet been reported. Therefore, we examined whether there were the anti-inflammatory properties of BV in a mouse model of OVA-induced skin inflammation.

$\mathrm{AD}$ has been reported to be characterized by hyperkeratosis, spongiosis, epidermal hyperplasia, and accumulation of lymphocyte and mast cell (34). Therefore, our study examined the effect of BV on the characteristics of AD. We measured epidermal thickness to assess the extent of hyperkeratosis, spongiosis, and epidermal hyperplasia. The markedly increased epidermal thickness in AD-like skin lesions by OVA has been shown to decrease through BV treatment. These results suggest that $\mathrm{BV}$ inhibits characteristic symptoms of AD in OVA-induced AD-like skin lesions.

Filaggrin, a major protein involved in the terminal differentiation of epidermal keratinocytes, has an important structural and functional role in the epidermis, which is influential in homeostasis of the skin (18). Variations in the filaggrin genotype, environmental factors, and skin microenvironment have been reported as major contributors to reduced levels of filaggrin (18). Filaggrin deficiency in AD patients is known to affect epidermal function and increase 


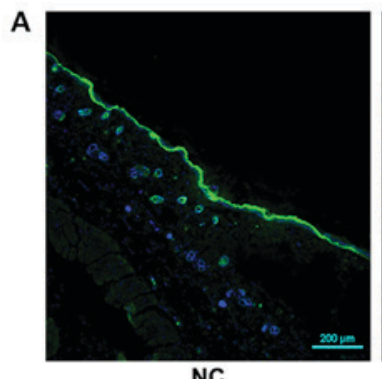

NC

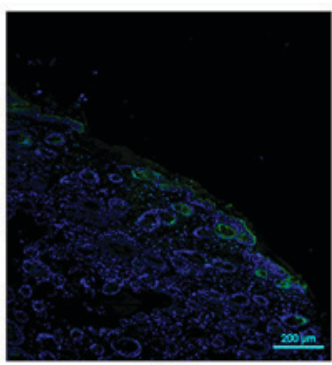

OVA

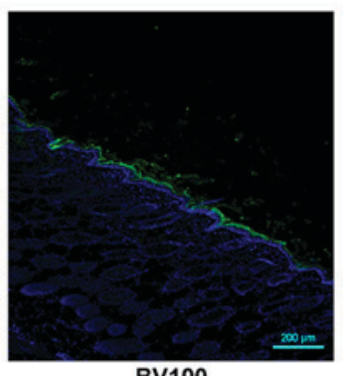

BV100

B

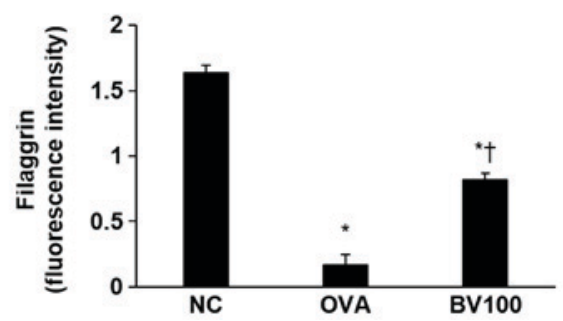

Figure 4. BV improves filaggrin deficiency by OVA. (A) Representative immunofluorescence images showing the improvement in filaggrin deficiency (stained with Alexa Fluor 488, green) following BV treatment. The nuclei were stained with Hoechst 33342 (blue). Magnification, x100; scale bar, $200 \mu \mathrm{m}$. (B) The fluorescence intensities of filaggrin were measured. ${ }^{\mathrm{P}}<0.05$ vs. NC group; ${ }^{\mathrm{P}}<0.05$ vs. OVA group. BV, bee venom; OVA, ovalbumin; BV group, OVA with BV treatment $(100 \mu \mathrm{g} / \mathrm{kg}) ; \mathrm{NC}$, normal control.

the risk of microbial infection or other atopic diseases $(18,35)$. Therefore, an attempt was made in the current study to determine whether or not BV treatment would reduce the symptoms of OVA-induced skin diseases. Cha et al (36) showed that the increase in filaggrin expression contributes to the maintenance and enhancement of the skin barrier function. Similarly, our study showed that the expression of filaggrin deficient by OVA-induced improved the expression of filaggrin deficient by BV treatment. Based on this fact, we suggested that BV has an effect that contributes to maintenance and enhancement of skin barrier function. Based on this fact, increased expression of filaggrin resulting from BV treatment was considered to contribute to the maintenance and enhancement of skin barrier function and was observed to contribute to inhibition of OVA-induced AD-like skin lesions.

Mast cells are generally known to contribute to allergic reactions, including atopic skin lesions (34), and are caused by FceRI-activated allergens that bind to serum IgE-FceRI complexes $(6,37)$. The mast cells, activated by immunologic stimulation, undergo degranulation and release inflammatory mediators, including histamine, chemokines, and cytokines (8). Therefore, low IgE levels and inactivation of the mast cells are important in the alleviation of AD. It was observed in the current study that $\operatorname{IgE}$ expression and the number of mast cells involved in the inflammatory responses decreased in the BV-treated, OVA-induced mice AD model. Several studies have shown that elevated levels of serum IgE and pro-inflammatory cytokines in AD have been reported $(38,39)$. In addition, pro-inflammatory cytokines, such as IL-1 $\beta$, IL-6, and TNF- $\alpha$, are known to induce the production of inflammatory mediators, such as histamine, and protease and chemotactic factors, in the mast cells (40). Pro-inflammatory cytokines are produced by inflammatory dendritic cells, i.e., differentiated mononuclear cells recruited during the acute AD phase. In our Previously, we demonstrated that inhibition of pro-inflammatory cytokines by
BV treatment in $P$. acnes-induced acne skin inhibited inflammatory skin disease (41). Similar to these results, BV treatment was demonstrated to reduce the expression of pro-inflammatory cytokines in OVA-induced AD-like skin lesions in the current study. These data suggest that it is likely that BV would be an effective treatment in AD-like skin disease.

It has been shown in several studies that pro-inflammatory cytokine expression, i.e., interferon (IFN)- $\gamma$, IL-4, IL-13, IL-17, and TSLP, increases in AD skin lesions $(42,43)$. TSLP, the keratinocyte-derived cytokine, has been shown to play an important role in the early stages of allergic inflammation. It was identified as a sensitive product of keratinocytes released before the lesion skin developed In AD patients (44). In addition, it has been shown to activator of sensory neurons that causes itching; a vital feature of atopic skin (45). Elsewhere, it was demonstrated that the down-regulation of TSLP improved AD (46). This was similar to the finding of the current study, where a reduction in TSLP expression was observed following BV treatment. Therefore, the modulation of AD-associated TSLP and cytokine expression could result in therapeutic efficacy. BV was demonstrated to suppress TSLP and pro-inflammatory cytokine expression in the OVA-induced, AD-like mice model in the present study. Therefore, the use of BV has considerable potential as an alternative AD therapy for AD lesions.

In addition, TSLP binds to the dendritic cell receptor and promotes the differentiation of CD4+ T cells into Th2 cells (14). CD4+ T cells are key components of allergic inflammatory disease. CD11b+ is a monocyte antigen that is expressed on many leukocyte surfaces, including granulocytes, monocytes, and macrophages (47). Thus, it was deemed worthwhile to investigate whether or not BV could alleviate AD-like skin lesions (especially allergic diseases) through an evaluation of CD4+ T and CD11b+ expression. In this study, it was confirmed that CD4+ T and CD11b+ expression was markedly decreased following BV treatment. Thus, BV 
treatment was demonstrated to have a therapeutic effect on AD-like inflammatory disease. It can be used to effectively maintain skin barrier function, prevent the destruction of the skin barrier, regulate proinflammatory cytokine levels, inhibit mast cell activation, and reduce inflammation.

BV has an anti-inflammatory effect on AD in this experiment. In previous study, melittin which is a major component of BV also showed an anti-inflammatory function for AD (28). It seems to be due to melittin, the main component of $\mathrm{BV}$. Thus, the pharmacological function of BV could be similar to that of melittin.

In conclusion, it was demonstrated in the present study that the administration of BV via intraperitoneal inoculation effectively suppressed the onset of atopic skin lesions. BV inhibited cytokines that are influential in $\mathrm{AD}$ via a decline in IgE, TNF- $\alpha$, and TSLP in vivo, and demonstrated effective anti-inflammatory activity. Therefore, we propose that BV is an alternative therapy for the treatment of anti-inflammatory AD. However, the exact anti-inflammatory mechanism of BV components requires further investigation.

\section{Acknowledgements}

Not applicable.

\section{Funding}

The present study was conducted with the support of the 'Cooperative Research Program for Agriculture Science and Technology Development (project no. PJ01316601)' Rural Development Administration, Republic of Korea.

\section{Availability of data and materials}

The datasets used or analyzed during the current study are available from the corresponding author on reasonable request.

\section{Authors' contributions}

HG, W-HK and K-KP conceived and supervised the study. HG, H-JA, J-YK, W-HK and M-GG performed the experiments. HG, SMH and JL performed the data analysis. HG and $\mathrm{K}-\mathrm{KP}$ drafted the manuscript. All authors discussed, revised and approved the manuscript.

\section{Ethics approval and consent to participate}

Animal care and all experimental procedures were approved by the Institutional Animal Care and Use Committee of Catholic University of Daegu (approval no. DCIAFCR-160428-1-Y) and experiments were performed in accordance with these institutional guidelines.

\section{Patient consent for publication}

Not applicable.

\section{Competing interests}

The authors declare that they have no competing interests.

\section{References}

1. Leung DY and Guttman-Yassky E: Deciphering the complexities of atopic dermatitis: Shifting paradigms in treatment approaches. J Allergy Clin Immunol 134: 769-779, 2014.

2. Schlapbach C and Simon D: Update on skin allergy. Allergy 69: 1571-1581, 2014.

3. Lim SJ, Kim M, Randy A, Nam EJ and Nho CW: Effects of Hovenia dulcis Thunb. extract and methyl vanillate on atopic dermatitis-like skin lesions and TNF- $\alpha / \mathrm{IFN}-\gamma$-induced chemokines production in $\mathrm{HaCaT}$ cells. J Pharm Pharmacol 68: 1465-1479, 2016

4. Galli SJ, Tsai M and Piliponsky AM: The development of allergic inflammation. Nature 454: 445-454, 2008.

5. Owen CE: Immunoglobulin E: Role in asthma and allergic disease: Lessons from the clinic. Pharmacol Ther 113: 121-133, 2007.

6. Kawakami T, Ando T, Kimura M, Wilson BS and Kawakami Y: Mast cells in atopic dermatitis. Curr Opin Immunol 21: 666-678, 2009.

7. Stone KD, Prussin C and Metcalfe DD: IgE, mast cells, basophils, and eosinophils. J Allergy Clin Immunol 125 (2 Suppl 2): S73-S80, 2010.

8. Kempuraj D, Castellani ML, Petrarca C, Frydas S, Conti P, Theoharides TC and Vecchiet J: Inhibitory effect of quercetin on tryptase and interleukin-6 release, and histidine decarboxylase mRNA transcription by human mast cell-1 cell line. Clin Exp Med 6: 150-156, 2006.

9. Leung DY, Boguniewicz M, Howell MD, Nomura I and Hamid QA: New insights into atopic dermatitis. J Clin Invest 113: 651-657, 2004.

10. Miazgowicz MM, Headley MB, Larson RP and Ziegler SF: Thymic stromal lymphopoietin and the pathophysiology of atopic disease. Expert Rev Clin Immunol 5: 547-556, 2009.

11. Ziegler SF: The role of thymic stromal lymphopoietin (TSLP) in allergic disorders. Curr Opin Immunol 22: 795-799, 2010.

12. Wilson SR, Thé L, Batia LM, Beattie K, Katibah GE, McClain SP, Pellegrino M, Estandian DM and Bautista DM: The epithelial cell-derived atopic dermatitis cytokine TSLP activates neurons to induce itch. Cell 155: 285-295, 2013.

13. Soumelis V, Reche PA, Kanzler H, Yuan W, Edward G, Homey B, Gilliet M, Ho S, Antonenko S, Lauerma A, et al: Human epithelial cells trigger dendritic cell mediated allergic inflammation by producing TSLP. Nat Immunol 3: 673-680, 2002 .

14. Watanabe N, Hanabuchi S, Soumelis V, Yuan W, Ho S, de Waal Malefyt R and Liu YJ: Human thymic stromal lymphopoietin promotes dendritic cell-mediated CD4+ T cell homeostatic expansion. Nat Immunol 5: 426-434, 2004.

15. Ebner S, Nguyen VA, Forstner M, Wang YH, Wolfram D, Liu YJ and Romani N: Thymic stromal lymphopoietin converts human epidermal Langerhans cells into antigen-presenting cells that induce proallergic T cells. J Allergy Clin Immunol 119: 982-990, 2007.

16. Wang Q, Du J, Zhu J, Yang X and Zhou B: Thymic stromal lymphopoietin signaling in CD4(+) T cells is required for TH2 memory. J Allergy Clin Immunol 135: 781-791.e3, 2015.

17. Kim BS, Siracusa MC, Saenz SA, Noti M, Monticelli LA, Sonnenberg GF, Hepworth MR, Van Voorhees AS, Comeau MR and Artis D: TSLP elicits IL-33-independent innate lymphoid cell responses to promote skin inflammation. Sci Transl Med 5: 170ra16, 2013.

18. Cabanillas B and Novak N: Atopic dermatitis and filaggrin. Curr Opin Immunol 42: 1-8, 2016.

19. Levin J, Friedlander SF and Del Rosso JQ: Atopic dermatitis and the stratum corneum: Part 1: The role of filaggrin in the stratum corneum barrier and atopic skin. J Clin Aesthet Dermatol 6: 16-22, 2013.

20. Son DJ, Lee JW, Lee YH, Song HS, Lee CK and Hong JT: Therapeutic application of anti-arthritis, pain-releasing, and anti-cancer effects of bee venom and its constituent compounds. Pharmacol Ther 115: 246-270, 2007.

21. Billingham ME, Morley J, Hanson JM, Shipolini RA and Vernon CA: Letter: An anti-inflammatory peptide from bee venom. Nature 245: 163-164, 1973.

22. Kwon YB, Lee HJ, Han HJ, Mar WC, Kang SK, Yoon OB, Beitz AJ and Lee JH: The water-soluble fraction of bee venom produces antinociceptive and anti-inflammatory effects on rheumatoid arthritis in rats. Life Sci 71: 191-204, 2002. 
23. Kwon YB, Lee JD, Lee HJ, Han HJ, Mar WC, Kang SK, Beitz AJ and Lee JH: Bee venom injection into an acupuncture point reduces arthritis associated edema and nociceptive responses. Pain 90: 271-280, 2001

24. Stieger M, Wüthrich B, Wyss S and Kopper E: Clinical picture and diagnosis of bee-venom allergy. A comparison between skin tests and RAST determinations. Hautarzt 29: 632-637, 1978 (In German).

25. Ip SW, Liao SS, Lin SY, Lin JP, Yang JS, Lin ML, Chen GW, Lu HF, Lin MW, Han SM and Chung JG: The role of mitochondria in bee venom-induced apoptosis in human breast cancer MCF7 cells. In Vivo 22: 237-245, 2008

26. Oršolić N: Bee venom in cancer therapy. Cancer Metastasis Rev 31: 173-194, 2012.

27. Park MH, Choi MS, Kwak DH, Oh KW, Yoon DY, Han SB, Song HS, Song MJ and Hong JT: Anti-cancer effect of bee venom in prostate cancer cells through activation of caspase pathway via inactivation of NF- $\mathrm{KB}$. Prostate 71: 801-812, 2011.

28. Kim WH, An HJ, Kim JY, Gwon MG, Gu H, Jeon M, Sung WJ, Han SM, Pak SC, Kim MK and Park KK: Beneficial effects of melittin on ovalbumin-induced atopic dermatitis in mouse. Sci Rep 7: 17679, 2017.

29. Han SM, Lee GG and Park KK: Acute dermal toxicity study of bee venom (Apis mellifera L.) in rats. Toxicol Res 28: 99-102, 2012.

30. Dombrowski Y, Peric M, Koglin S, Kaymakanov N, Schmezer V, Reinholz M, Ruzicka T and Schauber J: Honey bee (Apis mellifera) venom induces AIM2 inflammasome activation in human keratinocytes. Allergy 67: 1400-1407, 2012.

31. Kim JY, Lee WR, Kim KH, An HJ, Chang YC, Han SM, Park YY, Pak SC and Park KK: Effects of bee venom against Propionibacterium acnes-induced inflammation in human keratinocytes and monocytes. Int J Mol Med 35: 1651-1656, 2015.

32. Lee WR, Kim SJ, Park JH, Kim KH, Chang YC, Park YY, Lee KG, Han SM, Yeo JH, Pak SC and Park KK: Bee venom reduces atherosclerotic lesion formation via anti-inflammatory mechanism. Am J Chin Med 38: 1077-1092, 2010.

33. Han S, Lee K, Yeo J, Baek H and Park K: Antibacterial and anti-inflammatory effects of honeybee (Apis mellifera) venom against acne-inducing bacteria. J Med Plants Res 4: 459-464, 2010.

34. Lee YJ, Kim JE, Kwak MH, Go J, Kim DS, Son HJ and Hwang DY: Quantitative evaluation of the therapeutic effect of fermented soybean products containing a high concentration of GABA on phthalic anhydride-induced atopic dermatitis in IL-4/Luc/CNS-1 Tg mice. Int J Mol Med 33: 1185-1194, 2014.

35. van den Oord RA and Sheikh A: Filaggrin gene defects and risk of developing allergic sensitisation and allergic disorders: Systematic review and meta-analysis. BMJ 339: b2433, 2009.
36. Cha HY, Ahn SH, Cheon JH, Park SY and Kim K: Hataedock treatment has preventive therapeutic effects for atopic dermatitis through skin barrier protection in Dermatophagoides farinae-induced NC/Nga mice. J Ethnopharmacol 206: 327-336, 2017.

37. Amin K: The role of mast cells in allergic inflammation. Respir Med 106: 9-14, 2012.

38. Choi EJ, Debnath T, Tang Y, Ryu YB, Moon SH and Kim EK: Topical application of Moringa oleifera leaf extract ameliorates experimentally induced atopic dermatitis by the regulation of Th1/Th2/Th17 balance. Biomed Pharmacother 84: 870-877, 2016.

39. Liu FT, Goodarzi H and Chen HY: IgE, mast cells, and eosinophils in atopic dermatitis. Clin Rev Allergy Immunol 41: 298-310, 2011.

40. Sae-Wong C, Mizutani N, Kangsanant S and Yoshino S: Topical skin treatment with Fab fragments of an allergen-specific IgG1 monoclonal antibody suppresses allergen-induced atopic dermatitis-like skin lesions in mice. Eur J Pharmacol 779: 131-137, 2016.

41. An HJ, Lee WR, Kim KH, Kim JY, Lee SJ, Han SM, Lee KG, Lee CK and Park KK: Inhibitory effects of bee venom on Propionibacterium acnes-induced inflammatory skin disease in an animal model. Int J Mol Med 34: 1341-1348, 2014.

42. You CE, Moon SH, Lee KH, Kim KH, Park CW, Seo SJ and Cho SH: Effects of emollient containing bee venom on atopic dermatitis: A double-blinded, randomized, base-controlled, multicenter study of 136 patients. Ann Dermatol 28: 593-599, 2016.

43. Liu YJ: Thymic stromal lymphopoietin: Master switch for allergic inflammation. J Exp Med 203: 269-273, 2006.

44. Comeau MR and Ziegler SF: The influence of TSLP on the allergic response. Mucosal Immunol 3: 138-147, 2010.

45. Jeong HJ, Hong SH, Lee DJ, Park JH, Kim KS and Kim HM: Role of $\mathrm{Ca}(2+)$ on TNF-alpha and IL-6 secretion from RBL-2H3 mast cells. Cell Signal 14: 633-639, 2002.

46. Han NR, Moon PD, Kim HM and Jeong HJ: Tryptanthrin ameliorates atopic dermatitis through down-regulation of TSLP. Arch Biochem Biophys 542: 14-20, 2014.

47. Solovjov DA, Pluskota E and Plow EF: Distinct roles for the alpha and beta subunits in the functions of integrin alphaMbeta2. J Biol Chem 280: 1336-1345, 2005.

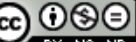

This work is licensed under a Creative Commons Attribution-NonCommercial-NoDerivatives 4.0 International (CC BY-NC-ND 4.0) License. 